\title{
Simulation of Hard Hand over (HHO) Mechanism in IEEE 802.16j Transparent Mode networks
}

\author{
D.Satish Kumar \\ Assistant Professor, \\ Kalaignar Karunanidhi Institute of Technology, \\ Coimbatore-641402, INDIA.
}

\author{
Dr.N.Nagarajan \\ Principal \\ Coimbatore Institute of Engineering and Technology \\ Coimbatore-641109, INDIA
}

\begin{abstract}
Two different relay modes are defined in IEEE 802.16j WIMAX standard: transparent mode and non-transparent mode. IEEE $802.16 \mathrm{j}$ standard supports mobile station mobility; it defines three kinds of hand over mechanism: hard handover, macro diversity handover (MDHO) and fast BS switching (FBSS)

In this paper we have simulated the hard hand over mechanism of IEEE 802.16j transparent mode networks using NCTUns Tool. We have used Adaptive modulation and coding scheme. The results show when the mobile station moves form base station 1 (BS1) to base station 2(BS2), it has to disconnect the original connection with BS1 before making a new connection with BS2. And also it shows that the throughput gradually decrease when the mobile station moves to NLOS.

Key-Words: - IEEE 802.16j, transparent mode, hand over mechanism, NCTUns, throughput etc
\end{abstract}

\section{INTRODUCTION}

IEEE802.16 standard was authorized in year 1999. An amendment 802.16-e (mobile WIMAX also called m-WIMAX) operates both in licensed and unlicensed band and was designed mainly to point to multipoint access. WIMAX (Worldwide interoperability for micro wave access technology) will be the most significant technology to date in making wireless access ubiquitous, as more free spectrum is opened up. IEEE 802.16-e Mobile WIMAX air interface adopts orthogonal frequency division multiple access (OFDMA) for improved multi-path performance in non Loss of sight (NLOS).

An amendment to the 802.16-e standard namely 802.16-j specifying relay station(RS) and multi-hop relay base station(MR-BS) approved in year 2006 supports Mobile multihop Relay networks.(MMR). It provides coverage extension to isolated area and throughput enhancement by specifying relay stations. This system enables mobile stations to communicate with a base station through intermediate relay station.

IEEE 802.16j operates in two modes, transparent mode and non transparent mode. Transparent mode is used to increase the capacity of BS. Non transparent mode is used to increase the coverage area of BS. A new scheme called as tunneling introduced in IEEE 802.16j networks, which is designed specially to leverage the inherent notion of aggregation in relay links.

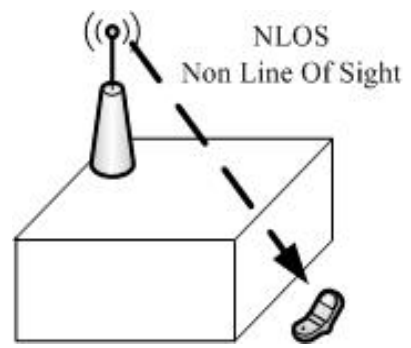

Fig 1: NLOS in $802.16 \mathrm{j}$
The base station would use the standard medium access control layer (MAC) a common interface that makes the networks interoperable to nearly instantaneously allocate uplink and downlink bandwidth to subscribers according to their needs. In IEEE 802.16j relay project, the RS adds MAC protocol to support multi-hop communication between RS, and also BS must support multiple RS. The above Fig 1 shows the NLOS (non Loss of sight) in IEEE 892.16 networks.

\section{IEEE 802.16J MAC LAYER.}

In MAC layer two different forwarding schemes are used, the tunnel based scheme and CID based scheme. The tunnel based scheme provides support for tunnels characterized by the unique CID, two specific end points and QOS parameters. The CID based scheme has no tunnels and does not support traffic aggregation; it only supports legacy management and transport connections.

MAC layer provides routing and path management. As 802.16j network comprises multi-hop paths between the BS and MS, the standard defines two approaches for path management, embedded and explicit path management. It also defines network entry management.

The MAC layer handover of IEEE $802.16 \mathrm{j}$ includes the following phases: 1) network topology acquisition before handover, which includes network topology advertisement, and MS scanning or association of neighbor BSs. 2) handover execution phase, which mainly includes cell reselection, handover initialization and handshake process, connection release, and target network reentry.

\section{HARD HAND OVER (HHO).}

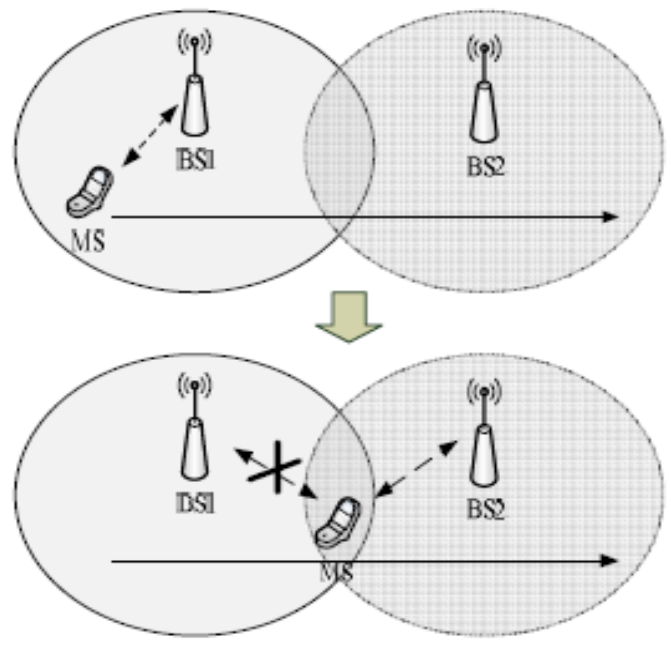

Fig 2: The hard handover mechanism. 
The hard hand over mechanism as shown in fig 2 uses the principle of break before make. This is the MS will break the connection with the original BS before making a new connection with another BS. Although it may lower the handover quality, an improved hand over mechanism must be used. When the MS moves from BS1 to BS2, it has to disconnect the original connection with BS1 before it can make a new connection with BS2. The figure 3 shows the simulation topology for the MS to communicate directly with BS. Here the BS operates in transparent mode.

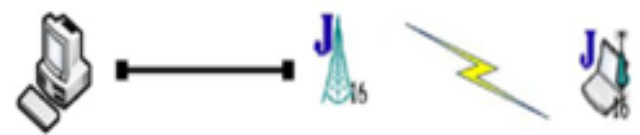

Fig 3: 802.16j MS connected to 802.16j BS

We have referred several papers and web sites solve hand over issues in IEEE 802.16j networks. To meet the paper length limitation, we listed below [6], [7], [8], [9], [10], and [11]

\section{HAND OVER PROCESS.}

The Hand over process is composed of several phases: network topology advertisement, Mobile station scanning, cell Reselection, Hand over decision and initiation, network reentry (ranging, reselection re-registration), and normal operation. The criteria for Hand over decision and initiation is , channel quality indicators such as CINR(Carrier to interface and noise Ration) or the signal strength can be exchanged and QOS is characterized by service level prediction of MS and BS. Other criteria such as bit error rate (BER), packet delay/jitter, service pricing, MS velocity; Ms Location can also be used.

Hands over stages are.

Stage-1: The MS collects information about BSs in the neighborhood.

Stage-2: While scanning MS seeks for suitable BS that is added in the diversity set.

Stage-3: Two types of report are send by MS, event triggered report and periodic reporting.

In event triggered report Ms Send reports after each measurement of Relative delay, round trip delay, receive signal strength report and CINR, but in periodic reporting reports are sending at periodic intervals.

Stage-4: Cell Reselection

Stage-5: MS Hand over is initialized based on the comparison of signal level of the current BS and new BS.

Stage-6: MS Network Re-entry consist of three stages: ranging (obtaining information about uplink channel), Re-authorization and Re-registration.

Stage-7: MS Normal operation;

\section{DEFINITION AND EXAMPLES.}

We first give simple definitions for key terms as they will be used in the rest of this paper:

- IEEE 802.16j: It is the standard for IEEE 802.16j Mobile WIMAX relay networks, It is an amendment to IEEE 802.16e2005

- TMR-BS: IT is the transparent mode mobile relay base station for IEEE 802.16j Mobile WIMAX relay networks.

- T-MS: It is the transparent mode mobile station, which is an wireless interface to TMR -BS.

- Hand over: It is a connection hand over from one BS1 to another BS2.

- T-RS: It is the transparent mode relay station, which is an interface to TMR -BS.
- OFDMA: It is modulation scheme called orthogonal Frequency division multiple access (OFDMA) used in IEEE 802.16j.

\section{SIMULATION SETUP.}

An IEEE 802.16j multi hop mobile WIMAX relay network is constructed using topology editor of NCtuns as shown in the below figure. A single host is connected with two 802.16j base stations through three bridges and three links as shown in the figure 4. Each Link band width is set as $50 \mathrm{Mbps}$. The BS1 and MS form a subnet 1, BS2 form subnet 2 .

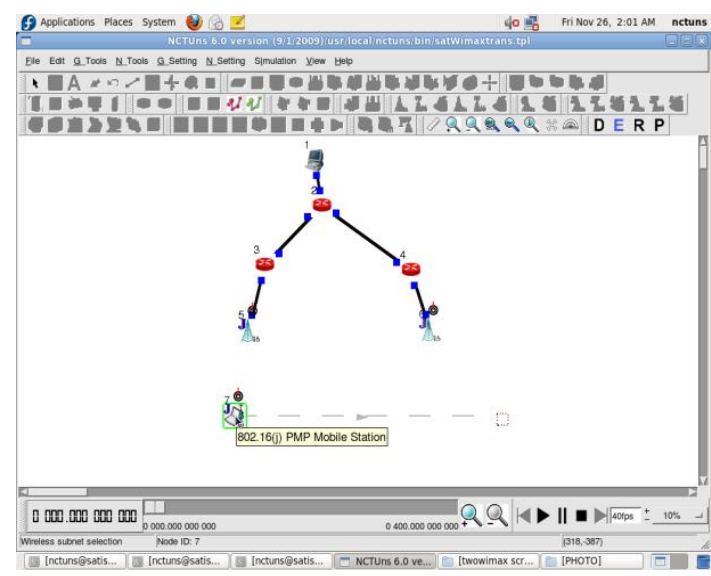

Fig 4: 802.16j PMP Mobile station

A single mobile station is connected to the base station 1(BS1) through wireless subnet. The speed of the MS is set as 20 $\mathrm{m} / \mathrm{sec}$, thus the MS will be moving along the horizontal path from point $(\mathrm{x} 1 \mathrm{y} 1)$ to point $(\mathrm{x} 2, \mathrm{y} 2)$ as shown in the figure. Here point $(x 1, y 1)$ is within the base coverage region of BS1, and point (x2.y2) is in the base coverage region of BS2.

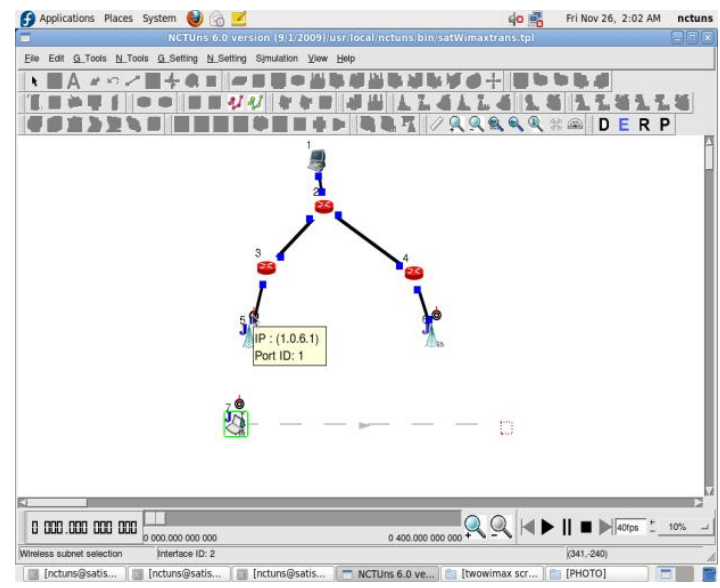

Fig 5: 802.16j base station (BS1)

The $802.16 \mathrm{j}$ base station 1 (BS1) is connected to the host and mobile station as shown above fig 5 . 


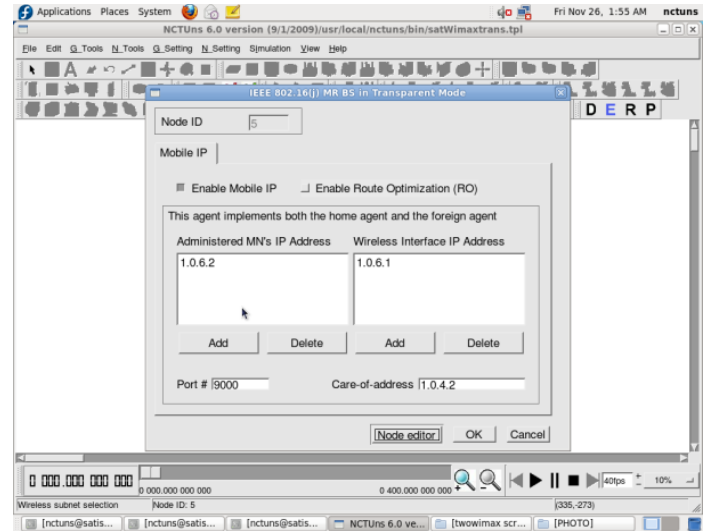

Fig 6: Base station 1 IP address

Figure 6 shows BS1 IP address for wired connection is set as 1.0.6.1 for wired connection to the link, and set as 1.0.7.1 for wireless connection to the MS.

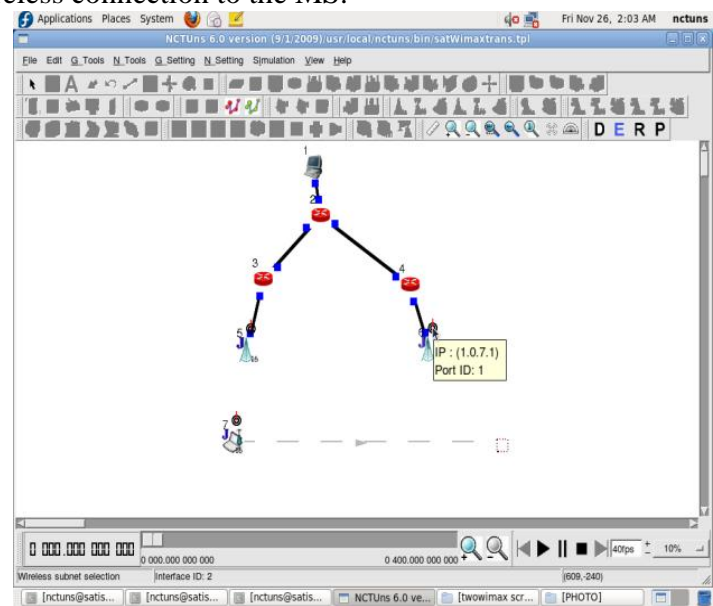

Fig 7: 802.16j WIMAX Base station (BS2)

The simulation time is set as

1. Simulation start time $=1 \mathrm{sec}$

2. MS Starting time $=3 \mathrm{sec}$

3. MS Stop time $=34 \mathrm{sec}$

4. Simulation close time $=400 \mathrm{sec}$.

Host channel parameters.

1. Bandwidth $=50 \mathrm{Mbps}$

2. Bit error rate $=0$

3. Propagation delay $=0.0$ Micro seconds.

4. $\quad$ IP address $=1.0 .1 .1$

5. Net mask $=255.255 .255 .0$

6. ARP protocol.

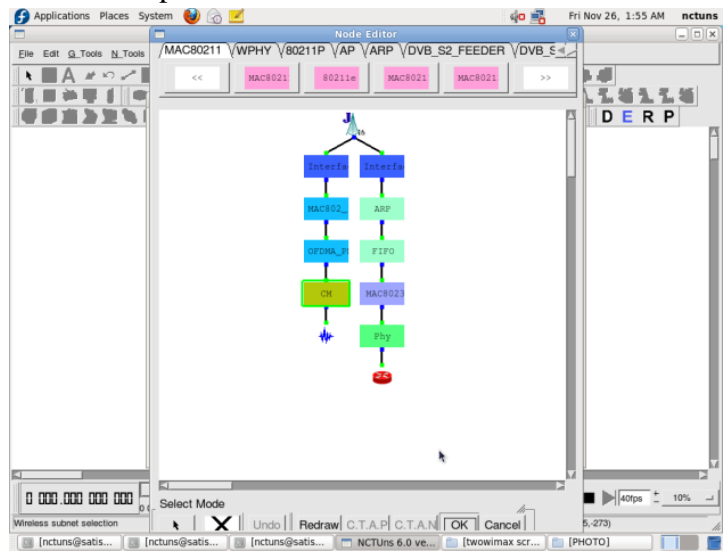

Fig 8: 802.16j BS node editor

802.16j Base station channel parameters.

1. Fading variance $=10$

2. Average building height $=10 \mathrm{~m}$

3. Average Building distance $=80 \mathrm{~m}$

4. Street width $=30 \mathrm{~m}$

5. Pass loss exponent $=2.0$

6. Shadowing standard deviation $=4.0$

7. Close in reference distance $(\mathrm{m})=1.0$

8. System loss $=1.0$

9. Antenna height $=30 \mathrm{~m}$

10. Ricean factor $(\mathrm{k})=10.0 \mathrm{db}$

OFDMA parameters for BS

1. Channel $\mathrm{ID}=5$

2. Frequency $=2300 \mathrm{MHz}$

3. Transmission power $=35 \mathrm{dbm}$

4. Receive sensitivity $=-99 \mathrm{dbm}$

802.16j Mobile station channel parameters

1. Frequency $=2300 \mathrm{MHz}$

2. Transmission power $=35 \mathrm{dbm}$

3. Receive sensitivity $=-99 \mathrm{dbm}$

4. Speed $=20 \mathrm{~m} / \mathrm{sec}$

Mobile station positions.

Initial Position $(\mathrm{x} 1, \mathrm{y} 1)=(133,453)$

Final position $(\mathrm{x} 2, \mathrm{y} 2)=(878,464)$

The node editor for $802.16 \mathrm{j}$ Base station is shown in the figure 8, it consists of various modules like ARP , FIFO, MAC8023, OFDMA interface and physical layer modules, the parameters of each module is set as shown above. The propagation channel mode can be set theoretical by specifying the parameters for path loss or fading model. In empirical channel model it defines 22 types of model. We have chosen empirical channel model.

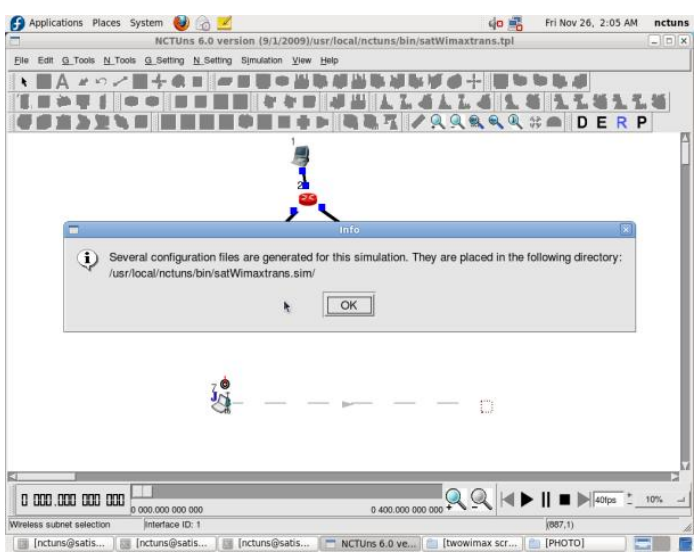

Fig 9: Running of simulation

Then the simulation is executed using as shown in below figure, all the related files of simulation is placed in the directory /usr/local/nctuns/bin/ as shown below, and all the log files are also placed in the same directory . 


\section{SIMULATION RESULTS.}

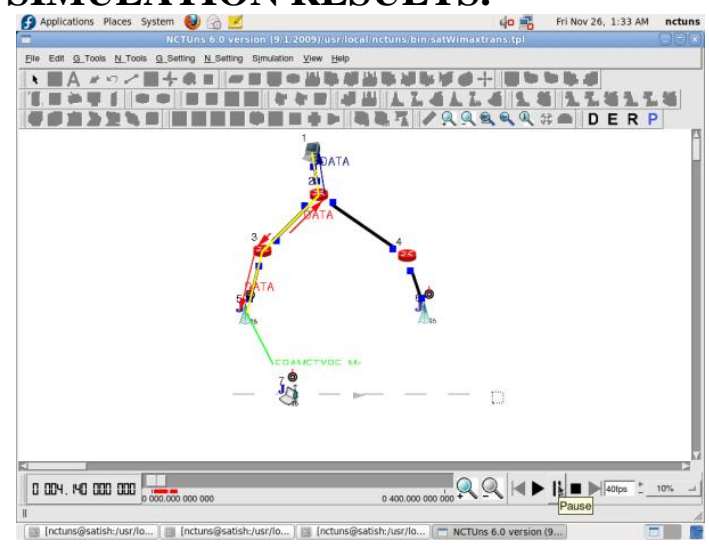

Fig 10: Initial transfer of packets through BS1

The above figure 10 shows the initial transfer of packets with the base station BS1. The mobile station is moving at a speed of $20 \mathrm{~m} / \mathrm{sec}$ form initial position towards the final position as specified in the path.

As the mobile station moves out of coverage region of BS1, handover occurs from BS1 to BS2, then the packets are transferred through BS2, the starting time of initial data transfer through BS2 is $3 \mathrm{sec}$, hand over occurs at $22 \mathrm{sec}$ and the starting time of initial data transfer through BS2 is $24 \mathrm{sec}$ and stiping time is $34 \mathrm{sec}$.

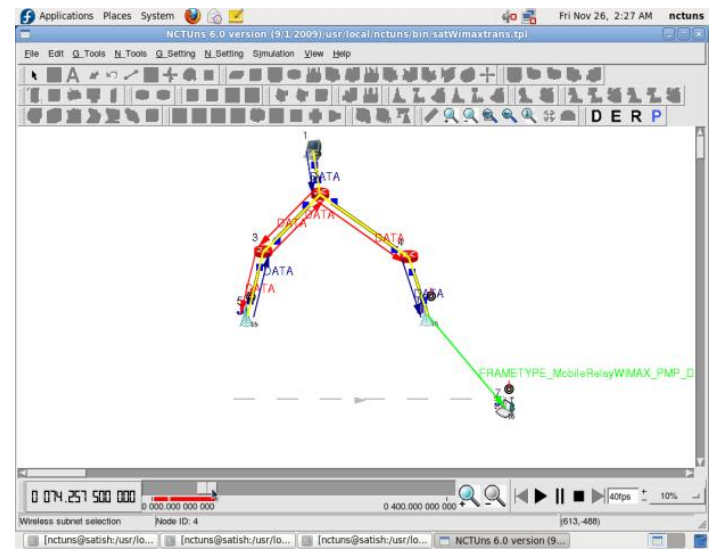

Fig 11: Hand over \& transfer of packets through BS2

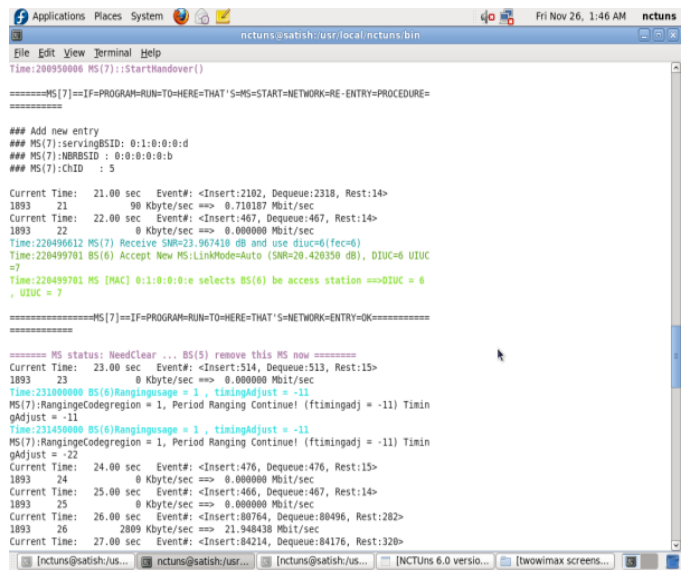

Fig 12: Hand over from BS1 to BS2 at time $22 \mathrm{sec}$

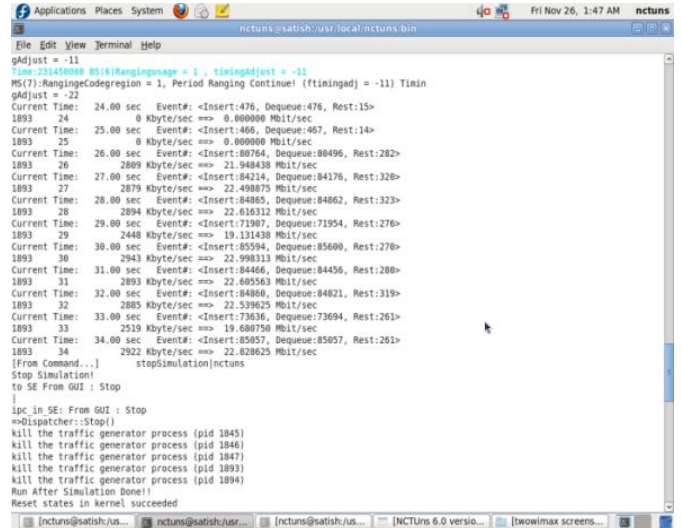

Fig 13: Packets transfer through BS2 at time $24 \mathrm{sec}$

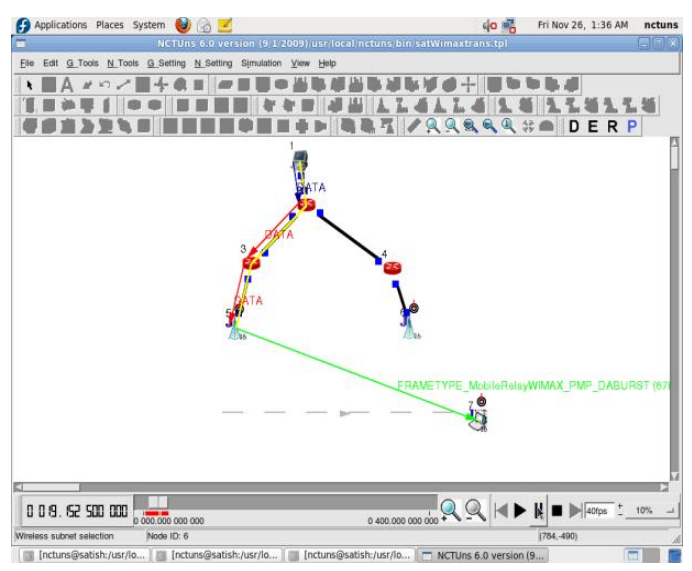

Fig 14: NLOS of BS1

The downlink throughput when the adaptive modulation and coding scheme is used is shown in below figure $15 \& 16$

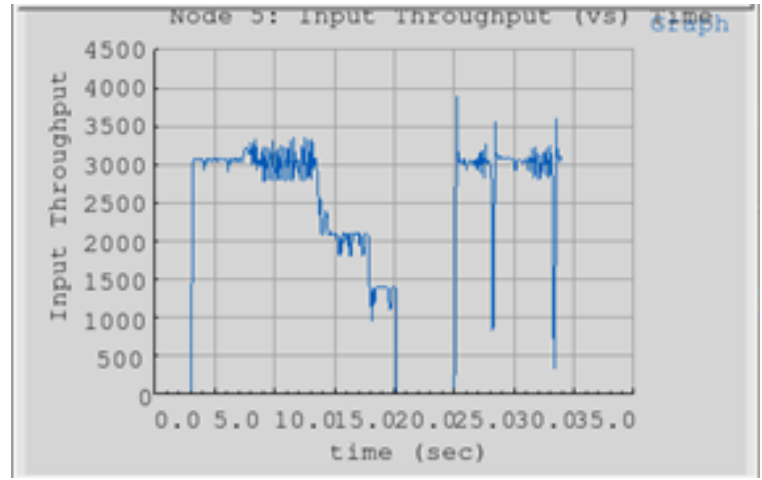

Fig 15: Downlink overall throughput (BS1 \& BS2) 


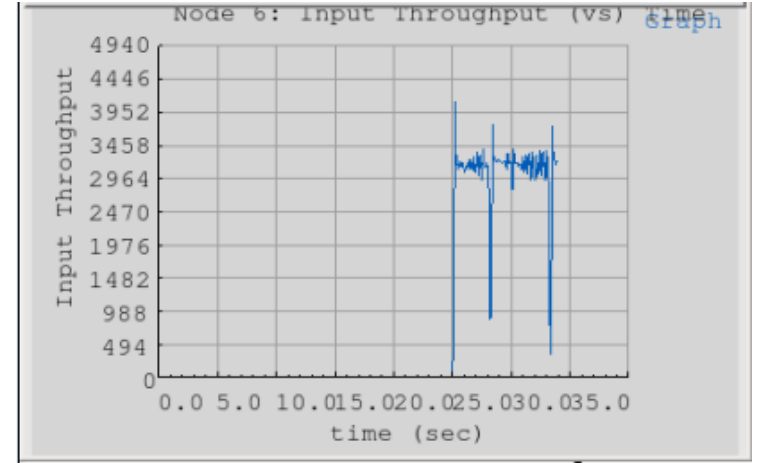

Fig 16: Downlink Base station 2 throughput (BS2)

The throughput is as shown above here the base station throughput is given, the overall through put shows that during the hand over and during switch to other base staion the throughput gradually decreases to zero and again after hand over it increases to maximum. As the hand over time is $4 \mathrm{sec}$, during that period the throughput will be zero.

\section{CONCLUSIONS AND FUTURE WORKS}

There is decrease in downlink throughput as the mobile station moves out of coverage area of Base station (BS1), and also the during the Hard hand over process no packets are transferred. The hand over time is $2 \mathrm{sec}$, after handover the packets are transferred through BS2. We are planning to define a hand over mechanism to increase the throughput and also to decrease the hand over time

\section{REFERENCES}

[1] IEEE Std 802.16-2009, "IEEE Standard for Local and Metropolitan Area Networks Part 16: Air Interface for Fixed Broadband Wireless Access Systems," 29 May 2009.

[2] Wang, S. Y. \& Lin, Y. B. (2005). "NCTUns Network Simulation and Emulation for Wireless Resource Management," Wiley Wireless Communications and Mobile Computing, Vol. 5, Issue 8, December, 899-916.

[3] NCTUns Tool for IEEE 802.16j Mobile WiMAX Relay Network Simulations, Shie-Yuan Wang*, Hsin-Yu Chen, and Shih-Wei Chuang Department of Computer Science National Chiao Tung University, Hsinchu, Taiwan.

[4] S.Y. Wang, H.Y. Chen, and S.W. Chuang, "NCTUns Tool for IEEE 802.16j Mobile WiMAX Relay Network Simulations," a chapter of the "Computer Science Research and Technology" book, (ISBN: 978-1-61728-688-9, published by Nova Science Publishers in 2009)

[5] S.M. Huang, Y.C. Sung, S.Y. Wang, and Y.B. Lin, "NCTUns Simulation Tool for WiMAX Modeling," Third Annual International Wireless Internet Conference, October 22 - 24, 2007, Austin, Texas, USA. (EI and ISI indexed, sponsored by ICST, ACM, EURASIP)

[6] A. George et al., "Protocols for Mobility Management in Heterogeneous Multihop Wireless Networks," Pervasive and Mobile Computing, vol. 4, no. 1, 2008, pp. 92-116.

[7] Lee D.H., Kyamakya K., Umondi J.P.. Fast handover algorithm for IEEE 802.16e Broadband wireless access system[C]. ISWPC 2006.

[8] WiMAX Forum: "A Technical Overview and Performance Evaluation," Mobile WiMAX - Part I, August 2006.

[9] WiMAX Community, "WiMAX fundamentals Quality of Service", June 2007.
[10] A. Singhrova and N. Prakash. A review of vertical handoff decision algorithm in heterogeneous networks. In International conference on mobile technology, applications, and systems, Singapore, Singapore, Sept. 2007.

[11] H. Izumikawa, Y. Kishi, and R. Nagareda. Novel WLAN coverage area estimation leveraging transition of cellular signal using multi-mode mobile terminal for heterogeneous networks. In IEEE Wireless Communications and Networking Conference, Budapest, Hungary, April 2009.

[12] P. Goyal and S. K. Saxena. A dynamic decision model for vertical handoffs across heterogeneous wireless networks. Proceedings of World Academy of Science, Engineering and Technology, 31(1):677-682, 2008.

[13] P. Li, X. Yi, and Y. Pan, "A seamless handover mechanism for IEEE 802.16e systems," IEEE Computer and Communications Societies Conference (INFOCOM), vol. 3, pp. 985-992, April 1995.

[14] Garg, A, Kin Choong Yow, "Determining the best network tohandover among various IEEE 802.11 and IEEE 802.16 networks by amobile device", Mobile Technology, Applications and Systems, 15-17 Nov. 2005, pp. 1- 6.

[15] Doo Hwan Lee, Kyandoghere Kyamakya and Jean Paul Umondi,"Fast Handover Algorithm for IEEE 802.16e Broadband WirelessAccess System", wireless Pervasive Computing, 2006 1St International Symposium on, 16-18 Jan. 2006, pp. 1-6.

[16] Sik Choi, Gyung-Ho Hwang, Taesoo Kwon, Ae-Ri Lim, andDong-Ho Cho, "Fast Handover Scheme for Real-Time DownlinkServices in IEEE 802.16e BWA System", Vehicular TechnologyConference (2005 IEEE 61st), June 2005, Volume 3, pp. 2028-2032.

[17] I. Akyildz, J. Xie, and S. Mohanty, "A Survey of Mobility Management in Next Generation All IP Based Wireless Systems," IEEE Wireless Commun., vol. 11, no. 4, 2004,pp. 16-27.

[18] The NCTUns tool. available at http://nsl.csie.nctu.edu.tw/nctuns.html.

[19] IEEE standard for local and metropolitan area networks, part 16: Air interface for ${ }^{-} \mathrm{xd}$ broadband wireless acess systems. June 2004

\section{AUTHORS}

D. Satish Kumar received his B.E., degree in Electrical and Electronics Engineering from Bharathiar University and M.E. degree in Computer Science and Engineering from Manonmaniam Sundharnar University respectively. $\mathrm{He}$ is currently working towards the $\mathrm{PhD}$ degree in Computer Science and Engineering in Anna University of Technology Coimbatore, Tamil Nadu INDIA. His research interests accumulate in the area of IEEE 802.16j Multi hop Mobile networks, Routing, WIMAX networks, Network management in wired Networks and Wireless Networks.

Dr.N.Nagarajan received his B.Tech and M.E. degrees in Electronics Engineering at M.I.T Chennai. He received his $\mathrm{PhD}$ in Faculty of information and communication engineering from Anna University, Chennai. He is currently working as Principal, Coimbatore Institute of Engineering and Technology, Coimbatore. He is member of board of study of faculty of information Technology at Anna University of technology, Coimbatore. His specialization includes optical, wireless Adhoc and sensor networks. He is guiding assorted research scholars in optical networks and wireless networks. 